renal stem cells (Figure 1). The failure of whole bone marrow to have this protective effect (12) may be due to the extremely small numbers of MSCs present in the bone marrow or the large numbers of inflammatory cells infused in this preparation. It remains unclear whether the protective effect of MSCs requires them to leave the bone marrow and transit through the renal circulation or whether these cells can exert protective effects from distant sites. Identifying the protective factor(s) and the signals that prompt MSCs to secrete it should now be a priority in our attempts to develop new therapeutic approaches for improving patient outcomes following acute renal failure.

Address correspondence to: Lloyd G. Cantley, Department of Internal Medicine, Yale University, 333 Cedar Street, Box 208029, New Haven, Connecticut 06520,
USA. Phone: (203) 785-7110; Fax: (203) 785-3756; E-mail: Lloyd.Cantley@yale.edu.

1. Poulsom, R., et al. 2001. Bone marrow contributes to renal parenchymal turnover and regeneration. J. Pathol. 195:229-235.

2. Gupta, S., Verfaillie, C., Chmielewski, D., Kim, Y., and Rosenberg, M.E. 2002. A role for extrarenal cells in the regeneration following acute renal failure. Kidney Int. 62:1285-1290.

3. Lin, F., et al. 2003. Hematopoietic stem cells contribute to the regeneration of renal tubules after renal ischemia-reperfusion injury in mice. J. Am. Soc. Nephrol. 14:1188-1199.

4. Kale, S., et al. 2003. Bone marrow stem cells contribute to repair of the ischemically injured renal tubule. J. Clin. Invest. 112:42-49. doi:10.1172/ JCI200317856.

5. Lagasse, E., et al. 2000. Purified hematopoietic stem cells can differentiate into hepatocytes in vivo. Nat. Med. 6:1229-1234.

6. Krause, D.S., et al. 2001. Multi-organ, multi-lineage engraftment by a single bone marrow-derived stem cell. Cell. 105:369-377.

7. Herzog, E.L., Chai, L., and Krause, D.S. 2003. Plasticity of marrow-derived stem cells [review]. Blood. 102:3483-3493.
8. Morigi, M., et al. 2004. Mesenchymal stem cells are renotropic, helping to repair the kidney and improve function in acute renal failure. J. Am. Soc. Nephrol. 15:1794-1804.

9. Herrera, M.B., et al. 2004. Mesenchymal stem cells contribute to the renal repair of acute tubular epithelial injury. Int. J. Mol. Med. 14:1035-1041.

10. Duffield, J. 2005. Restoration of tubular epithelial cells during repair of the postischemic kidney occurs independently of bone marrow-derived stem cells. J. Clin. Invest. 115:1743-1755. doi:10.1172/JCI22593.

11. Togel, F., et al. 2005. Administered mesenchymal stem cells protect against ischemic acute renal failure through differentiation-independent mechanisms. Am. J. Physiol. Renal Physiol. doi:10.1152/ ajprenal.00007.2005.

12. Lin, F., Moran, A., and Igarashi, P. 2005. Intrarenal cells, not bone marrow-derived cells, are the major source for regeneration in postischemic kidney. J. Clin. Invest. 115:1756-1764. doi:10.1172/JCI23015.

13. Javazon, E.H., Beggs, K.J., and Flake, A.W. 2004. Mesenchymal stem cells: paradoxes of passaging. Exp. Hematol. 32:414-425.

14. Oliver, J.A., Maarouf, O., Cheema, F.H., Martens, T.P., and Al-Awqati, Q. 2004. The renal papilla is a niche for adult kidney stem cells. J. Clin. Invest. 114:795-804. doi:10.1172/JCI200420921.

\title{
Harlequin ichthyosis unmasked: a defect of lipid transport
}

\author{
Alain Hovnanian \\ Department of Medical Genetics and INSERM U563, Purpan Hospital, Toulouse, France.
}

\begin{abstract}
Harlequin ichthyosis (HI) - the most severe form of keratinizing disorders, often lethal in the neonatal period - is characterized by a profound thickening of the keratin skin layer, a dense "armor"-like scale that covers the body, and contraction abnormalities of the eyes, ears, and mouth. In this issue of the JCI, Akiyama et al. report that mutations in ABCA12 caused defective lipid transport that significantly impacted normal development of the skin barrier (see the related article beginning on page 1777). Lipid secretion was recovered after corrective $A B C A 12$ gene transfer into patient keratinocytes. These results should allow for early prenatal diagnosis of $\mathrm{HI}$ and lend hope to the possibility of a specific treatment for this devastating disorder.
\end{abstract}

Waring pointed to what is believed to be the first harlequin fetus described in the US in the diary of Reverend Oliver Hart in 1750 (1). Harlequin ichthyosis (HI) is believed to be inherited in an autosomalrecessive manner, and affected newborn infants are encased in "armor"-like thick, yellow plates of scales with deep red fissuring. The skin is pulled tight such that the face loses its normal appearance and

Nonstandard abbreviations used: HI, harlequin ichthyosis.

Conflict of interest: The author has declared that no conflict of interest exists.

Citation for this article: J. Clin. Invest. 115:1708-1710 (2005). doi:10.1172/JCI25736. appears frog-like, with eversion of the eyelids (ectropion) and lips (eclabion) and flattening of the ears and nose. The extremities are swollen due to constriction by massive thickening of the skin. Liveborn infants usually die within the first days of life from respiratory, infectious, and/or dehydration-related complications. Some patients treated with retinoids, synthetic derivatives of vitamin A, have survived and subsequently develop severe ichthyosis. The cause of HI, however, has remained elusive, and late prenatal diagnosis has until now relied on electron microscopic examination of tissue sampled by invasive fetal skin biopsy.

\section{Lipid transport: a likely suspect}

Members of the ABCA subclass of the large $A B C$ transporter protein family bind ATP for the active transport of lipids across cell membranes against a concentration gradient. $A B C A 1$ has been shown to be the causative gene in Tangier disease, a disorder of cholesterol transport between liver and other tissues (2-5), while mutations in $A B C A 4$ (expressed exclusively in photoreceptors of the eye for the transport of retinol) cause Stargardt disease, recessive retinitis pigmentosa, or cone-rod dystrophy, in which the abnormal accumulation of retinoids results in the development of macular dystrophy and loss of central vision (6-8).

Lipid processing in the skin is essential for the protective function of the stratum corneum, the most external layer of the epidermis (9). Corneocytes, attached to each other by corneodesmosomes and embedded in intercellular lipid lamellae, form a cornified layer that acts as a barrier between the internal and external environment for bodily defense. The lipid lamellae are derived from lamellar granules, the 
A

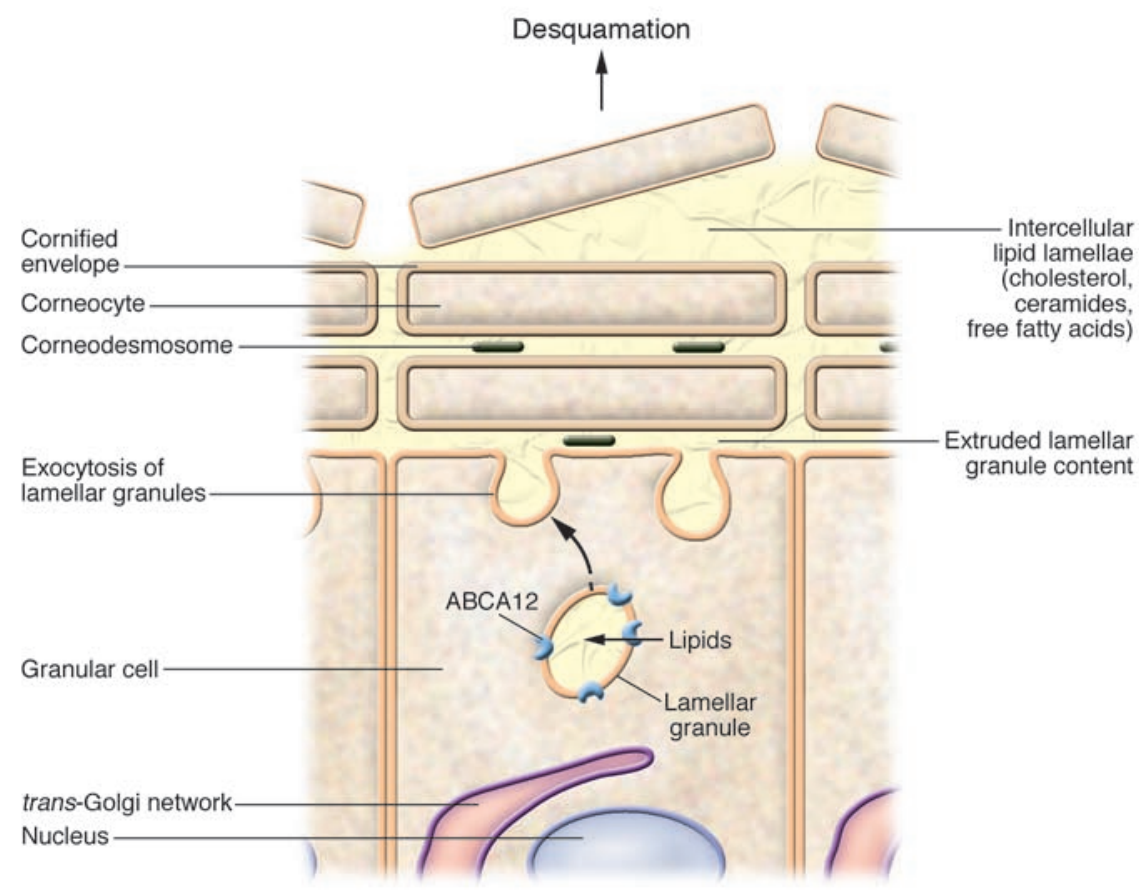

B

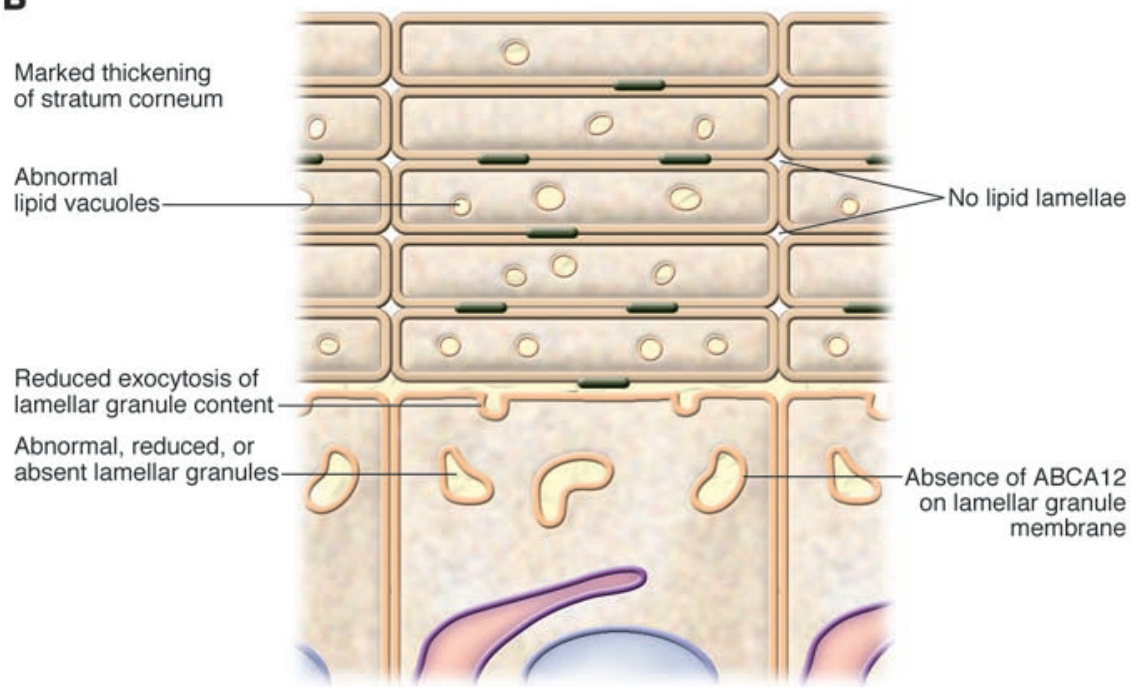

major lipid-rich organelles present in epidermal granular cells, which originate from the trans-Golgi network. Lamellar granules contain polar lipids (cholesterol sulfate, phospholipids, sphingomyelin, and glucosylceramides) that are the precursors of the intercellular lipids of the stratum corneum. Along with lipids, lamellar granules also transport lipid-processing enzymes and proteases and their inhibitors, all of which play a role in barrier permeability and the control of the desquamation process (10). Lipid-processing enzymes

\section{Figure 1}

Mutations in lipid transporter ABCA12 cause HI. (A) In the granular layers of healthy skin, the ABCA12 lipid transporter transfers lipids from the cytosol into lamellar granules where lipid-processing enzymes, proteases, and protease inhibitors are also concentrated. At the granular layer-stratum corneum interface, the lamellar granules fuse with the cell membrane and discharge their content into the intercellular lamellae. Complex enzymatic reactions lead to modifications of the lipid composition of the intercellular space (cholesterol, ceramides, free fatty acids) that provide a very effective water-permeability barrier. Corneocytes detach from each other in the superficial layers of the stratum corneum as a result of finely regulated proteolytic cleavage of corneodesmosomes. (B) In the skin of $\mathrm{HI}$ patients, the absence of ABCA12 prevents the transfer of lipids into lamellar granules, which themselves are abnormally shaped, reduced in number, or absent. As a result, exocytosis of lamellar granule content is reduced and intercellular lipid lamellae are absent. Abnormal lipid-containing vacuoles form in the cytoplasm of the corneocytes. The stratum corneum is remarkably thickened and does not desquame. 
Defects in lipid-processing enzymes have previously been shown to cause several other forms of ichthyosis. These include defects in: (a) the steroid sulfatase in $\mathrm{X}$-linked recessive ichthyosis (12); (b) $\beta$-glucocerebrosidase in Gaucher disease (13); (c) sphingomyelinase in Niemann-Pick disease (14); (d) fatty aldehyde dehydrogenase in Sjögren-Larsson syndrome (15); (e) lipoxygenase-3 and $12 \mathrm{R}$-lipoxygenase in autosomal-recessive congenital ichthyosis (16); and (f) CGI58 in Chanarin-Dorfman syndrome (17). Although none of these ichthyoses are as severe as HI, they illustrate that a major role for lipid abnormalities exists in the pathophysiology of ichthyosis.

\section{Mutations in $A B C A 12$ revealed}

In this issue of the JCI, Akiyama et al. (18) demonstrate that HI is caused by loss-offunction mutations in $A B C A 12$, which codes for a lamellar granule membrane protein involved in lipid transport (Figure 1A). Together with the knowledge that mutations in $A B C A 1$ and $A B C A 4$ cause Tangier disease and Stargardt disease, respectively, this most recent discovery further supports a pivotal role for ABCA class lipid transporters in cellular homeostasis and sheds light on the importance of lipid processing in the development and maintenance of the epidermal barrier.

The observation of lamellar granule abnormalities in epidermal keratinocytes of HI patients led Akiyama et al. (18) to test the hypothesis that a defect in a major lamellar granule protein could be defective in this disease. The authors drew a parallel between $A B C A 3$, which encodes a lamellar granule membrane protein essential for alveolar surfactant lipid transport and secretion in alveolar lung cells (19), and $A B C A 12$, which harbors missense mutations in autosomal-recessive congenital ichthyosis (20). The authors hypothesized that mutations in $A B C A 12$, more deleterious than those causing autosomal-recessive congenital ichthyosis, may underlie $\mathrm{HI}-$ and their hypothesis was correct. They demonstrate that 5 distinct mutations in $A B C A 12$ resulted in truncation or deletion of highly conserved regions of the ABCA12 protein, which disrupts lipid transport (Figure 1B). Interestingly, Kelsell and colleagues very recently used a completely different genomic approach, based on homozygosity mapping using single-nucleotide-polymorphism chip technology, to also identify $A B C A 12$ as the causative gene in $\mathrm{HI}(21)$.

As a consequence of impaired ABCA12 function, Akiyama et al. (18) found that lamellar granules were not properly formed and therefore the lipids essential for stratum corneum formation (such as glucosylceramide) were abnormally processed, diffusely distributed, and abnormally secreted or not secreted at all. The lack of lipid lamellae formation in the intercellular space resulted in abnormal barrier formation and extraordinary thickening of the stratum corneum (Figure 1B).

\section{Implications for therapy and prenatal diagnosis}

Akiyama et al. (18) also showed that genetic correction of $A B C A 12$ deficiency by gene transfer in patients' keratinocytes restored normal glucosylceramide cell distribution and lamellar granule formation. This result raises the possibility of $\mathrm{HI}$ treatment using systemic administration of functional peptides with ABCA12-like properties or $A B C A 12$ gene delivery approaches undertaken either prior to or after birth.

This discovery of the role of ABCA12 in $\mathrm{HI}$ reveals a major role of lipid transport in the formation of the skin barrier and its function. This is a very elegant illustration of adaptive evolution to terrestrial life, involving 2 closely related lipid transporters, ABCA3 and ABCA12, which are essential for the production of alveolar surfactant and lipid lamellae in the stratum corneum, respectively. At birth, while $\mathrm{ABCA} 3$ prevents the lungs from collapsing, ABCA12 protects the skin from external aggressions and water loss. Loss of ABCA12 expression results in the most severe dysregulation of cornification in humans, covering the newborn infant in a lethal type of armor. Not only will these findings dramatically improve our ability to offer mutational screening and early DNA-based prenatal diagnosis of HI, but they will also allow for the development of new and specific therapeutic approaches.

Address correspondence to: Alain Hovnanian, Department of Medical Genetics and INSERM U563, Purpan Hospital, BP3028, 31024 Toulouse Cedex 3, France. Phone: 33-562-74-45-00; Fax: 33-562-7445-58; E-mail: alain.hovnanian@toulouse. inserm.fr.
1. Waring, J.J. 1932. Early mention of a Harlequin fetus in America. Am. J. Dis. Child. 43:442.

2. Bodzioch, M., et al. 1999. The gene encoding ATP-binding cassette transporter 1 is mutated in Tangier disease. Nat. Genet. 22:347-351.

3. Brooks-Wilson, A., et al. 1999. Mutations in ABC1 in tangier disease and familial high-density lipoprotein deficiency. Nat. Genet. 22:336-345.

4. Rust, S., et al. 1999. Tangier disease is caused by mutations in the gene encoding ATP-binding cassette transporter 1. Nat. Genet. 22:352-355.

5. Remaley, A.T., et al. 1999. Human ATP-binding cassette transporter 1 (ABC1): genomic organization and identification of the genetic defect on the original Tangier disease kindred. Proc. Natl. Acad. Sci. U. S. A. 96:12685-12690.

6. Martinez-Mir, A., et al. 1998. Retinitis pigmentosa caused by a homozygous mutation in the Stargardt disease gene ABCR. Nat. Genet. 18:11-12.

7. Rozet, J.M., et al. 1999. Mutations of the retinal specific ATP binding transporter gene (ABCR) in a single family segregating both autosomal recessive retinitis pigmentosa RP19 and Stargardt disease: evidence of clinical heterogeneity at this locus. J. Med. Genet. 36:447-451.

8. Cremers, F.P., et al. 1998. Autosomal recessive retinitis pigmentosa and cone-rod dystrophy caused by splice site mutations in the Stargardt's disease gene ABCR. Hum. Mol. Genet. 7:335-362.

9. Madison, K.C. 2003. Barrier function of the skin: "la raison d'etre" of the epidermis [review]. J. Invest. Dermatol. 121:231-241.

10. Elias, P.M. 2004. The epidermal permeability barrier: from the early days at Harvard to emerging concepts. J. Invest. Dermatol. 122:xxxvi-xxxix.

11. Milner, M.E., O'Guin, W.M., Holbrook, K.A., and Dale, B.A. 1992. Abnormal lamellar granules in harlequin ichthyosis. J. Invest. Dermatol. 99:824-829.

12. Webster, D., France, J.T., Shapiro, L.J., and Weiss, R. 1978. X-linked ichthyosis due to steroid-sulphatase deficiency. Lancet. 1:70-72.

13. Sidransky, E., Tsuji, S., Martin, B.M., Stubblefield, B., and Ginns, E.I. 1992. DNA mutation analysis of Gaucher patients. Am. J. Med. Genet. 42:331-336

14. Schmuth, M., et al. 2000. Permeability barrier disorder in Niemann-Pick disease: sphingomyelinceramide processing required for normal barrier homeostasis. J. Invest. Dermatol. 115:459-466.

15. De Laurenzi, V., et al. 1996. Sjogren-Larsson syndrome is caused by mutations in the fatty aldehyde dehydrogenase gene. Nat. Genet. 12:52-57.

16. Jobard, F., et al. 2002. Lipoxygenase-3 (ALOXE3) and $12(\mathrm{R})$-lipoxygenase (ALOX12B) are mutated in non-bullous congenital ichthyosiform erythroderma (NCIE) linked to chromosome 17p13.1. Hum. Mol. Genet. 11:107-113.

17. Lefevre, C., et al. 2001. Mutations in CGI-58, the gene encoding a new protein of the esterase/ lipase/thioesterase subfamily, in Chanarin-Dorfman syndrome. Am. J. Hum. Genet. 69:1002-1012.

18. Akiyama, M., et al. 2005. Mutations in lipid transporter ABCA12 in harlequin ichthyosis and functional recovery by corrective gene transfer. J. Clin. Invest. 115:1777-1784. doi:10.1172/JCI24834.

19. Yamano, G., et al. 2001. ABCA3 is a lamellar body membrane protein in human lung alveolar type II cells. FEBS Lett. 508:221-225.

20. Lefevre, C., et al. 2003. Mutations in the transporter ABCA12 are associated with lamellar ichthyosis type 2. Hum. Mol. Genet. 12:2369-2378.

21. Kelsell, D.P., et al. 2005. Mutations in ABCA12 underlie the severe congenital skin disease harlequin ichthyosis. Am. J. Hum. Genet. 76:794-803. 